\title{
Anticipating intracranial hypertension before it can harm: Optic nerve sonography to monitor intracranial pressure after stroke
}

\author{
P. Baumgartner ${ }^{1}$, A.R. Luft ${ }^{1}$, S. Wegener ${ }^{1}$
}

'Department of Neurology, University Hospital Zurich and University of Zurich, Switzerland

\section{Background and Aims}

\section{Methods}

Intracranial Hypertension $(\mathrm{IH})$ is a life-threatening The goal of this study is to implement optic nerve complication in large vessel ischemic stroke (A/B). sonography (ONS) as an easy, non-invasive method to Mortality in this condition is up to $80 \%$ if treated measure ICP and detect IH after stroke. ONS utilizes the conservatively, thus fast diagnosis of $\mathrm{IH}$ and surgical fact that the optic nerve sheath, which is readily visualized referral for hemicraniectomy is imperative. Since invasive by transorbital ultrasound, is filled with spinal fluid and intracranial pressure (ICP) monitoring devices are not grows in diameter according to ICP. To perform ONS, the always feasible, patients are usually followed based on patient is placed in a position $20^{\circ}$ supine to horizontal. The clinical symptoms. However, medications or comorbidities ultrasound probe is then placed on the closed eyelid of stroke complicate clinical IH assessment. Therefore, without pressure to avoid any damage to the eye (C). readily available, non-invasive methods to early detect IH ONSD is then measured $3 \mathrm{~mm}$ behind the globe (D).

in stroke patients are needed.

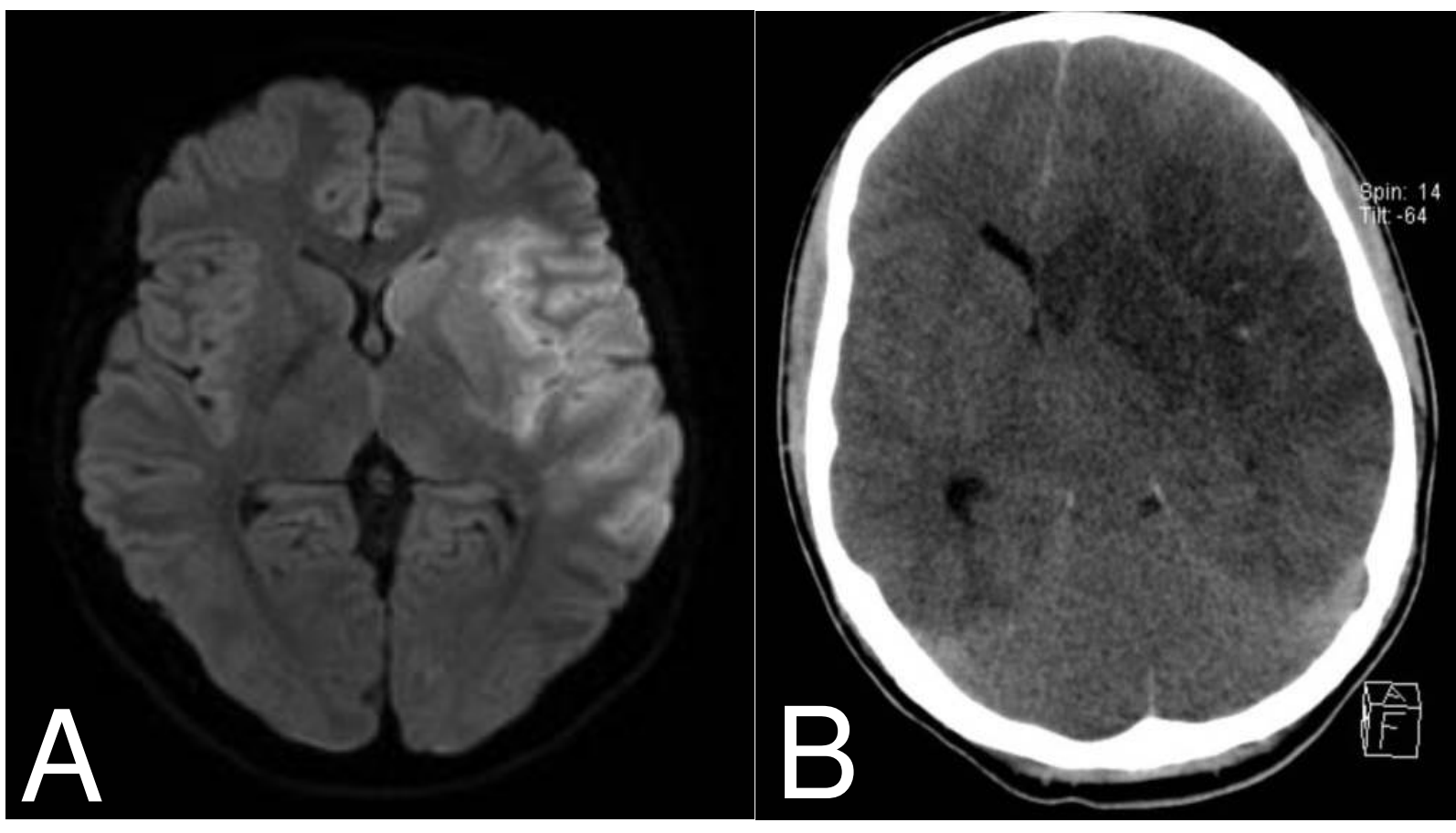

(A) Diffusion weighted MRI (DWI) of a 53y old patient with acute aphasia and right sided hemiparesis 4.5 hours after symptom onset. The left M1 could not be reanalyzed. (B): CT scan 2 days later shows large brain edema, clinically the patient had a new decrease in GCS.

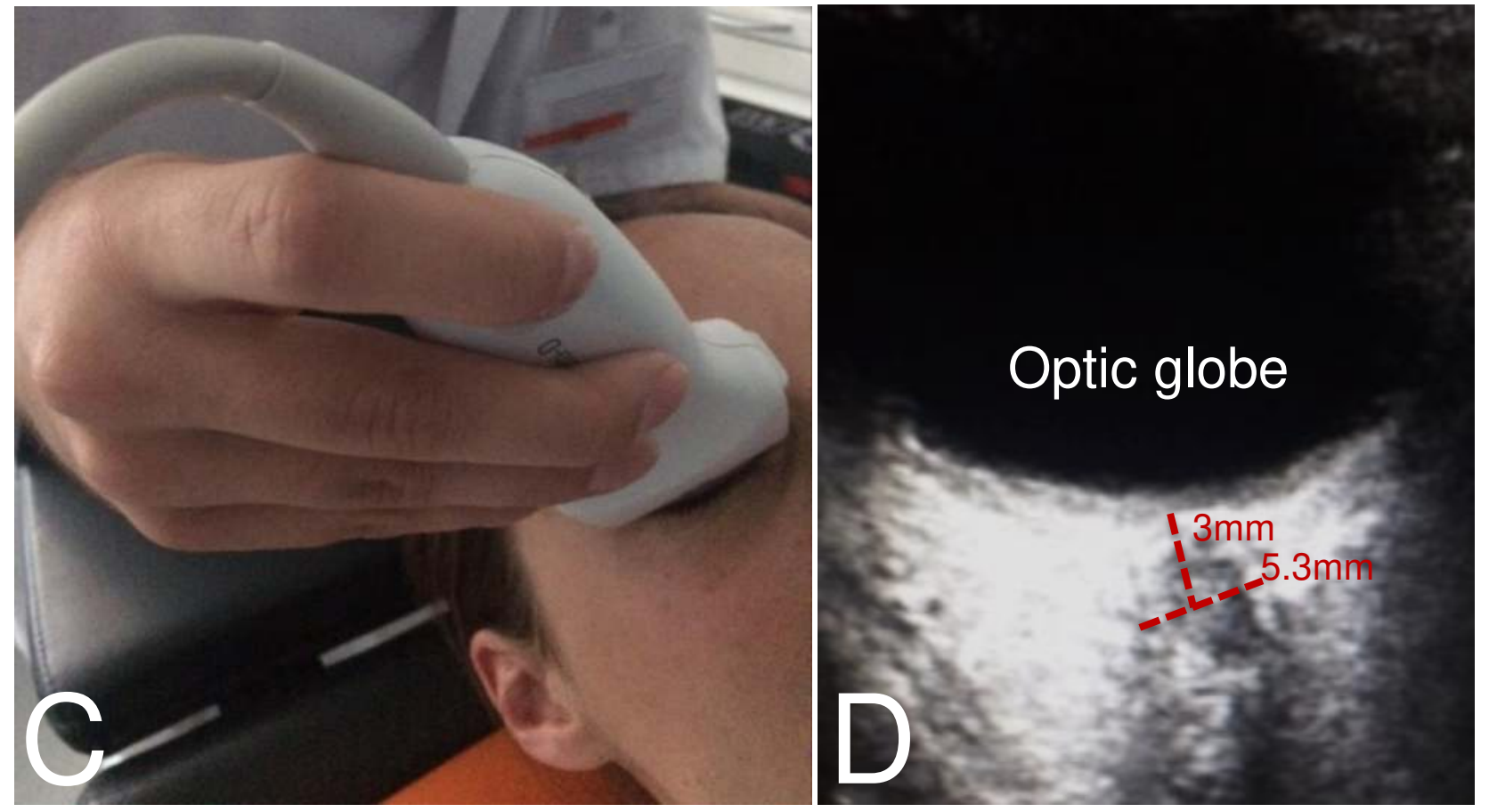

(C) ONS is performed in a patient by placing a $10 \mathrm{MHZ}$ linear transducer lightly upon the eyelid. (D): ONSD diameter measurement is performed $3 \mathrm{~mm}$ behind the optic globe. Three measurements per eye are usually averaged to reduce variability.

\section{Results}

We are planning a single-center, prospective observer study, where ONSD will be repeatedly assessed in patients with occlusion of the internal carotid artery (ICA) and/or the proximal segment (M1) of the middle cerebral artery up to 120 hours after stroke onset (E). GCS and NIHSS are obtained every 6 hours in clinical routine, and will be used along with neuroimaging (CT/MRI) to confirm IH. Cut-off values for ONSD that best predict the presence of $\mathrm{IH}(\mathrm{F})$ will then be determined by a receiver operating characteristic (ROC) curve. Sensitivity, specificity and positive predictive value of this threshold to predict IH after stroke will be calculated. The study is supported through the «Young Talents in Clinical Research» beginner grant of the Swiss academy of medical sciences (SAMS) and the Bangerter foundation. Own preliminary data show that ONSD is indeed increased ipsilateral to stroke $(G)$.

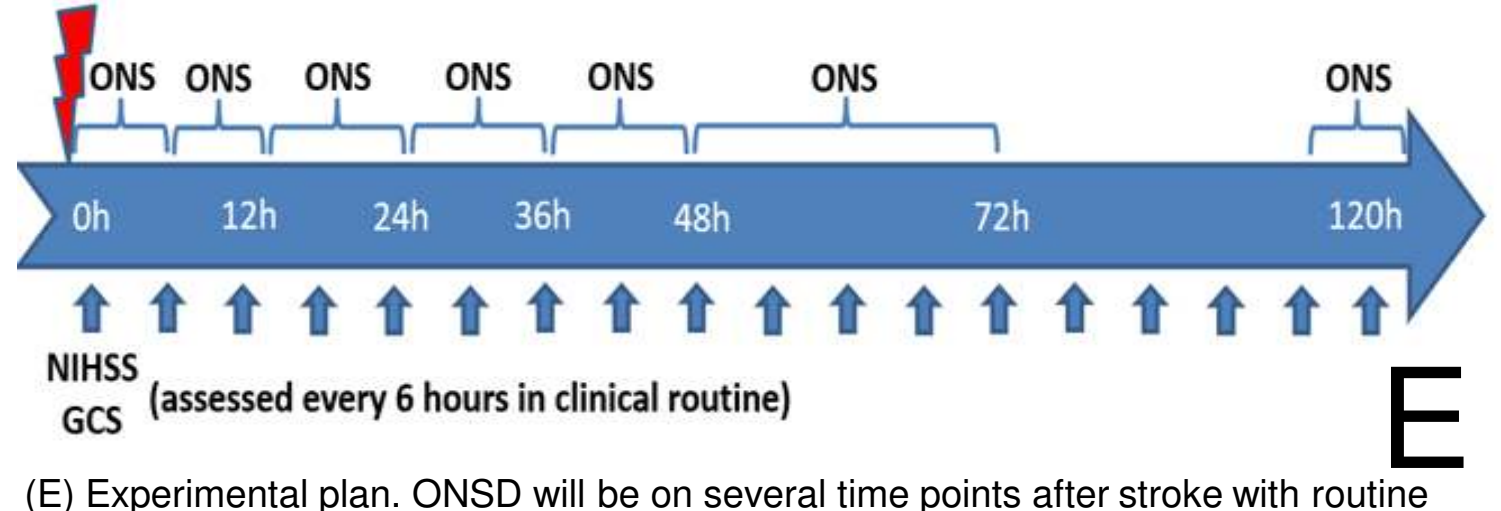
clinical assessment. Routine Neuroimaging will also be analysed to confirm $\mathrm{IH}$. (1)

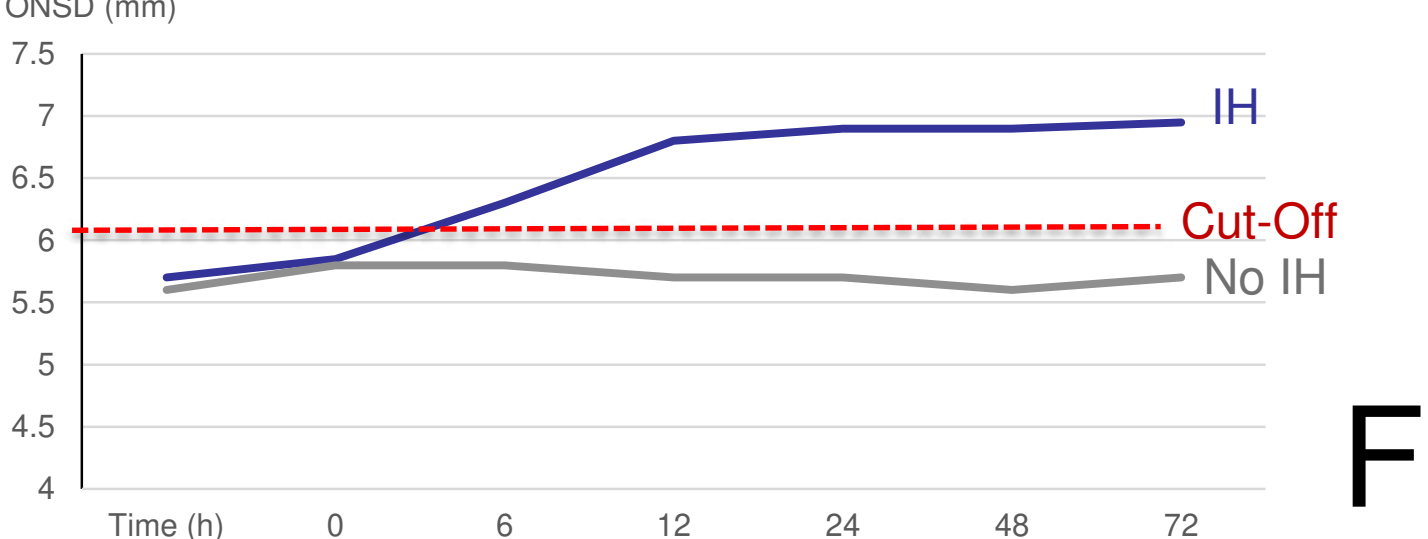

\section{Conclusion}

If ONSD thresholds can be established to detect IH early after stroke, it could be further tested in a clinical trial to aid in decision making for hemicraniectomy in stroke patients.

Philipp Baumgartner University Hospital of Zurich, Department of Neurology philipp.baumgartner@usz.ch 